\title{
Blast Coating of Superelastic NiTi Wire with PTFE to Enhance Wear Properties
}

\author{
Conor F. Dunne ${ }^{1} \cdot$ Kevin Roche ${ }^{2} \cdot$ Barry Twomey $^{2} \cdot$ Darel Hodgson $^{3} \cdot$ \\ Kenneth T. Stanton ${ }^{1}$
}

Published online: 21 April 2015

(C) ASM International 2015

\begin{abstract}
This work investigates the deposition of polytetrafluoroethylene (PTFE) onto a superelastic NiTi wire using an ambient temperature-coating technique known as CoBlast. The process utilises a stream of abrasive $\left(\mathrm{Al}_{2} \mathrm{O}_{3}\right)$ and a coating medium (PTFE) sprayed simultaneously at the surface of the substrate. Superelastic NiTi wire is used in guidewire applications, and PTFE coatings are commonly applied to reduce damage to vessel walls during insertion and removal, and to aid in accurate positioning by minimising the force required to advance, retract or rotate the wire. The CoBlast coated wires were compared to wire treated with PTFE only. The coated samples were examined using variety of techniques: X-ray diffraction (XRD), microscopy, surface roughness, wear testing and flexural tests. The CoBlast coated samples had an adherent coating with a significant resistance to wear compared to the samples coated with PTFE only. The XRD revealed that the process gave rise to a stress-induced martensite phase in the NiTi which may enhance mechanical properties. The study indicates that the CoBlast process can be used to deposit thin adherent coatings of PTFE onto the surface of superelastic NiTi.
\end{abstract}

Keywords Biocompatibility $\cdot \mathrm{NiTi} \cdot$ Materials $\cdot$ Shape memory - Shape memory films - Shape memory stents . Twinning

Kenneth T. Stanton

kenneth.stanton@ucd.ie

1 UCD School of Mechanical and Materials Engineering, University College Dublin, Belfield, Dublin 4, Ireland

2 ENBIO Ltd., Nova UCD, Belfield Innovation Park, Dublin 4, Ireland

3 Nitinol Technology, Inc., 740-D Sierra Vista Ave., Mountain View, CA 94043, USA

\section{Introduction}

NiTi-based alloys have been adopted for numerous biomedical applications such as orthodontic arch wires, guidewires and vascular stents due to their biocompatibility, superelastic (SE) and shape memory (SM) properties [1]. NiTi alloys possess relatively good biocompatibility and corrosion resistance due to the formation of a surface oxide composed mainly of $\mathrm{TiO}_{2}$.

When used in guidewire applications, a highly lubricous surface is desirable to reduce damage to vessel walls during insertion and removal [2], and to aid in accurate positioning by minimising the force required to advance, retract or rotate the wire [3]. The most common method for conferring a lubricous surface is to coat the wire with a hydrophobic polymer. Polytetrafluoroethylene (PTFE) is the most widely used coating for this purpose and there are a variety of PTFE-coated wires in the market [4].

This paper investigates a new method, CoBlast ${ }^{\mathrm{TM}}$, for depositing a PTFE coating onto superelastic NiTi wire. It is an ambient temperature process developed to address the problems associated with high temperature-coating techniques, such as the formation of unwanted phases, amorphization of the coating and poor adherence of the coating [5-14]. The coating produced with CoBlast is composed of separated particles firmly embedded within the substrate instead of the laminar structure typical of other coating techniques. This produces a unique level of adhesion and flexibility, and in many cases, eliminates the need for preor post-processing steps such as cleaning, roughening, priming, baking or curing. PTFE is an excellent example of a coating that traditionally requires a complex multistep process to bond it to a substrate, but is readily deposited in one step using CoBlast. NiTi is an excellent example of a 
substrate that is traditionally difficult to coat due to thermal sensitivity, but is readily coated using CoBlast without losing its unique properties.

CoBlast is a one-step process utilising a co-incident stream of abrasive and a coating particles to modify the substrate surface. The standard process uses a single nozzle driven by compressed air to apply a pre-blended combination of abrasive and coating. The abrasive roughens the surface while simultaneously disrupting the passivating oxide layer of the substrate and exposing the reactive metal. The coating particles then react with the exposed reactive metal to form an intimate chemical bond [7-11, 15-17]. The adhesion of the coating to the substrate is due to a combination of tribo-chemical bond formation and mechanical interlock between the dopant and the metal substrate [9]. Strong adhesion of PTFE to metals has been reported previously, in spite of the chemical inertness of PTFE. This has been attributed to fragmentation of the PTFE molecular chain due to mechanical stresses and subsequent carbon-metal bonding [18].

Previous studies have investigated the deposition of bioceramics onto titanium to enhance bioactivity [7-11, 15-17]. The process has been shown to exhibit a shot peen effect resulting in a severely deformed surface layer with twin formation and gross deformation of the grains in Grade 2 titanium. The formation of the deformed surface layer is beneficial as the associated compressive strength in the surface layer improves fatigue performance-a key property for orthopaedic medical devices, for example [7, 8, 19, 20].

Severe plastic deformation of NiTi has been investigated using various methods such as shot peening, high pressure torsion, cold-rolling, equal angular extrusion and laser shot peening, resulting in the formation of deformation-induced martensite (DIM) and amorphization [21-26]. The formation of DIM is associated with highly dense twinning and gives rise a work hardened layer and compressive residual stress that may enhance fatigue performance [25, 27].

\section{Materials and Methods}

\section{Materials}

Superelastic NiTi wire (Grade SE508, NDC, USA) with a diameter of $0.75 \mathrm{~mm}$ was used as the substrate. Five wires were coated per set. Also five NiTi $15 \times 15 \mathrm{~mm}$ coupons of $1 \mathrm{~mm}$ thickness were coated per set. The NiTi coupons were used in the surface characterisation of the coating and substrate using X-ray diffraction, surface roughness measurements and wear tests. PTFE (Zonyl 1300MP, Dupont, USA) was used as the coating media. Two particle sizes of alumina (White Saftigrit, $<50$ and $<90 \mu \mathrm{m}$, Guyson USA) were used to assess the influence of the blast media on the coating process. Figure 1 shows the particle images.

\section{Sample Preparation}

The NiTi wire and coupons were ultrasonically cleaned in isopropanol to remove any contaminants prior to surface modification. The NiTi wire was fed at $10 \mathrm{~mm} / \mathrm{s}$ through a circular array of 3 nozzles arranged around the wire axis. The coating medium (PTFE) and blast media $(<50$ or $<90 \mu \mathrm{m} \mathrm{Al}_{2} \mathrm{O}_{3}$ ) were sprayed simultaneously in the same stream at the NiTi wire with a jet pressure of $45 \mathrm{psi}$, at a nozzle displacement distance of $10 \mathrm{~mm}$ from the wire. Figure 2 show the schema of the wire-coating process.

The flat NiTi coupons were placed on a stationary tray. The coating medium (PTFE) and blast media $(<50$ or $<90 \mu \mathrm{m} \mathrm{Al}_{2} \mathrm{O}_{3}$ ) were sprayed simultaneously with a jet pressure of $40 \mathrm{psi}$, at a height of $30 \mathrm{~mm}$ from the substrate with a nozzle travel speed of $13 \mathrm{~mm} / \mathrm{s}$ and a raster offset of
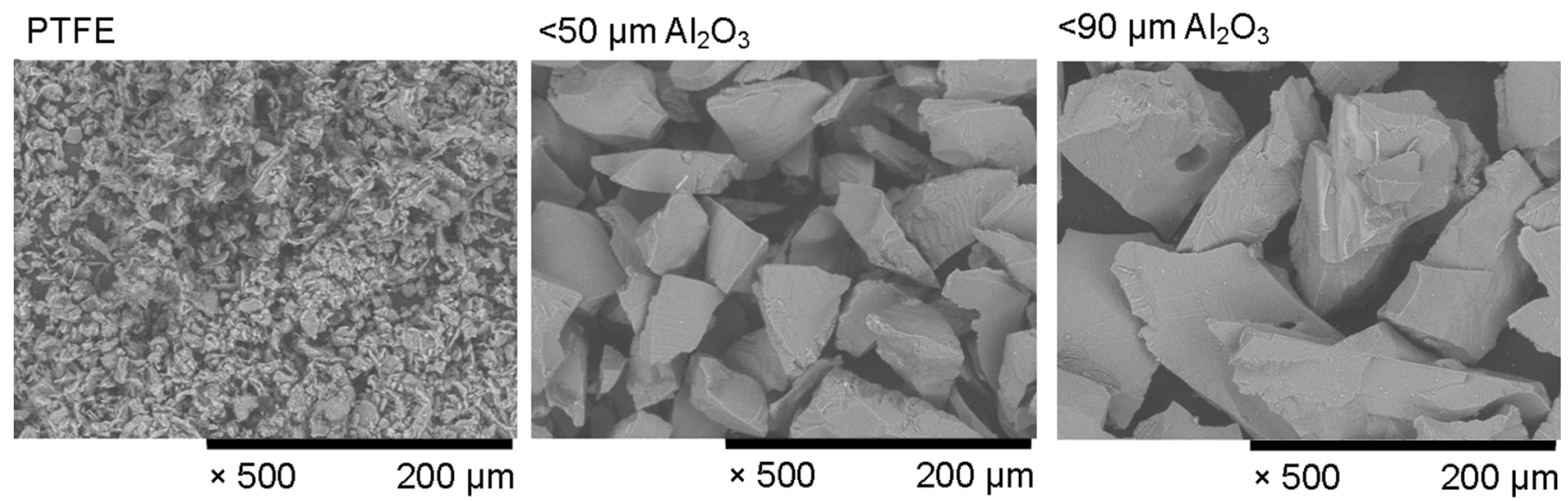

Fig. 1 SEM images of PTFE, $<50$ and $<90 \mu \mathrm{m} \mathrm{Al}_{2} \mathrm{O}_{3}$ powders 
Fig. 2 Schema of wire-coating process

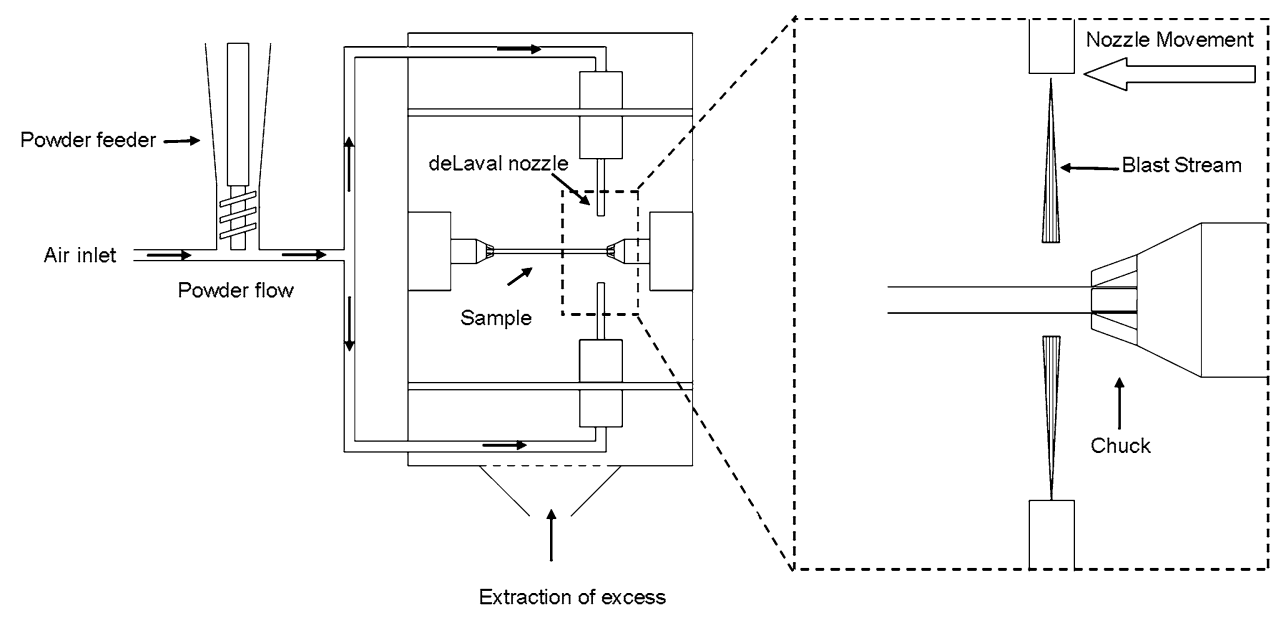

powder
Table 1 Substrate type and assigned code

\begin{tabular}{llll}
\hline Name & Blast medium & Coating medium & Substrate code \\
\hline NiTi (as-supplied) & - & - & $\mathrm{S}$ \\
NiTi (PTFE/ $\left.<50 \mu \mathrm{m} \mathrm{Al}_{2} \mathrm{O}_{3}\right)$ & $<50 \mu \mathrm{m} \mathrm{Al}_{2} \mathrm{O}_{3}$ & PTFE & A50P \\
NiTi (PTFE/ $\left.<90 \mu \mathrm{m} \mathrm{Al}_{2} \mathrm{O}_{3}\right)$ & $<90 \mu \mathrm{m} \mathrm{Al} \mathrm{O}_{3}$ & PTFE & A90P \\
NiTi (PTFE only) & - & PTFE & SP \\
NiTi $\left(<50 \mu \mathrm{ml}_{2} \mathrm{O}_{3}\right)$ & $<50 \mu \mathrm{m} \mathrm{Al}_{2} \mathrm{O}_{3}$ & - & A50 \\
NiTi $\left(<90 \mu \mathrm{m} \mathrm{Al}_{2} \mathrm{O}_{3}\right)$ & $<90 \mu \mathrm{m} \mathrm{Al}_{2} \mathrm{O}_{3}$ & - & A90 \\
\hline
\end{tabular}

$3.3 \mathrm{~mm}$. To act as a control, coupons and wires were prepared by blasting PTFE only, $<50 \mu \mathrm{m} \mathrm{Al}_{2} \mathrm{O}_{3}$ only and $<90 \mu \mathrm{m} \mathrm{Al} \mathrm{O}_{2} \mathrm{O}_{3}$ only. For clarity, a code has been assigned to each of the substrate types. The substrates and their associated code are shown in Table 1.

\section{Surface Characterisation}

X-ray diffraction was carried out on the NiTi coupons using a Siemens D500 diffractomer with rotating stage (Munich, Germany). A $\mathrm{Cu} \mathrm{K} \alpha$ radiation source was used with $\lambda=1.54056 \AA$. The spectra were measured using PTFE feedstock powder and also the flat monolithic coated substrates to assess the influence of the CoBlast treatment on the PTFE crystallinity. The analysis was carried out over the angular range of $10^{\circ}-90^{\circ}(2 \theta)$. All XRD scans were carried out with a resolution of $0.04^{\circ}(2 \theta)$ and a sampling time of $3 \mathrm{~s}$ per step. Silicon was used as an internal standard to account for non-linear peak shift.

The surface roughness of the coated NiTi coupons was measured using a Taylor-Hobson Pneumo Form Talysurf Series 2 (Leicester, UK). Four measurements per each of the five samples were used to determine the average roughness $\left(R_{\mathrm{a}}\right)$. Analysis of variance (ANOVA) and a subsequent Tukey's post hoc test were performed to determine whether the difference in roughness between the as-supplied NiTi and the coated NiTi substrates was statistically significant. A $P$ value $<0.05$ was deemed to be significant.

A Hitachi TM-1000 scanning electron microscope (SEM) (Toronto, Canada) in backscatter mode was used to examine the surfaces of the coupons after treatment.

Cross-sections of the NiTi coupons and wire were prepared in order to assess the coating thickness and examined using a Leica MEF4 M (Wetzlar, Germany) optical microscope with reflected light. To prepare the cross-sections for analysis, the samples were mounted in a polyester resin, then ground and polished to a $0.04 \mu \mathrm{m}$ finish, rinsed with water and isopropanol, respectively, and dried with a stream of warm air.

\section{Heat Treatment}

To investigate the thermal stability of martensite formed during the blasting process, substrates blasted with $<90 \mu \mathrm{m}$ $\mathrm{Al}_{2} \mathrm{O}_{3}$ only were heated for $1 \mathrm{~h}$ in a Carbolite furnace and cooled in air. Substrates were heated to 50, 100, 150, 200, 250 and $300{ }^{\circ} \mathrm{C}$ and examined using XRD as described in "Surface Characterisation" section. 


\section{Wear Test}

Wear testing was performed using a modified version of ASTM G133-95 using a custom-built tester. A load of $9.6 \mathrm{~N}$ was applied using a tungsten carbide pin with a diameter of $3.175 \mathrm{~mm}$ and a relative sliding speed of $3.4 \mathrm{~cm} /$ $\mathrm{s}$. The track length was $12 \mathrm{~mm}$ and the number of cycles was 5000, giving a total sliding length of $60 \mathrm{~m}$. The wear loss was calculated using Eq. 1,

Wear loss $=L\left[r^{2} \sin ^{-1}\left(\frac{d}{2 r}\right)-\left(\frac{d}{4}\right)\left(4 r^{2}-d^{2}\right)^{\frac{1}{2}}\right]$

where $L=$ wear track length, $r=$ pin end radius, $d=$ wear track width assuming that there were no significant losses to the pin. Five measurements of wear track width were made per track.

\section{Coating Adhesion}

The coating adhesion to the wire samples was examined by performing fracture and flex tests according to ISO 11070 and examining the surface at $1.5 \times$ magnification for cracks in the substrate or flaking of the coating. For the fracture test, the wire was wrapped around a mandrel of $6 \mathrm{~mm}$ diameter, then removed and examined. For the flex test, the wire was bent into and ' $S$ ' shape between two mandrels of diameter $13 \mathrm{~mm}$ with $1.4 \mathrm{~mm}$ gap between the mandrels. The wire was then straightened and bent again another 19 times, then examined. The coating was also examined using SEM and compared to a wire that was not subjected to the test.

\section{Results and Discussion}

The XRD traces of the coupons, Fig. 3, showing CoBlasted PTFE samples (A50P and A50P) exhibit intense PTFE peaks indicating good coating coverage/thickness, while the SP trace exhibits a weak PTFE peak indicating poor coating coverage/thickness. The coated samples exhibited peaks associated with NiTi which are present due to the underlying NiTi substrate due to the thin PTFE layer deposited $(<10 \mu \mathrm{m})$. The coated samples also exhibit peaks associated with deformation-induced martensite formed due to severe plastic deformation of the NiTi. This appears to be the B19' monoclinic martensite phase as it exhibits the characteristic $(001)$ reflection at $19^{\circ} 2 \theta$. The trace also displays strong (002) and (020) reflections at $39.2^{\circ} 2 \theta$ and $43.9^{\circ} 2 \theta$ respectively. The $(012)$ reflection at $45.1^{\circ} 2 \theta$ is very weak, however this should be most pronounced, this demonstrates that there is significant texturing of the matrix [23]. The blasting process has a stabilising effect on martensite, introducing dislocations and vacancies that increase the frictional resistance to austenite/martensite phase boundary movement inhibiting the reverse transformation and increasing the temperature at which martensite is stable [28]. The martensite phase becomes unstable above $300{ }^{\circ} \mathrm{C}$ when there is sufficient thermal energy to allow dislocation movement and annihilation allowing the back transformation of martensite to austensite to occur, Fig. 4.

The surface topography was examined using contact profilometry and SEM analysis. SEM of the surface of the coupons, Fig. 5, show the A50P and A90P substrates
Fig. 3 XRD patterns of the PTFE powder, S, SP, A50P and A90P

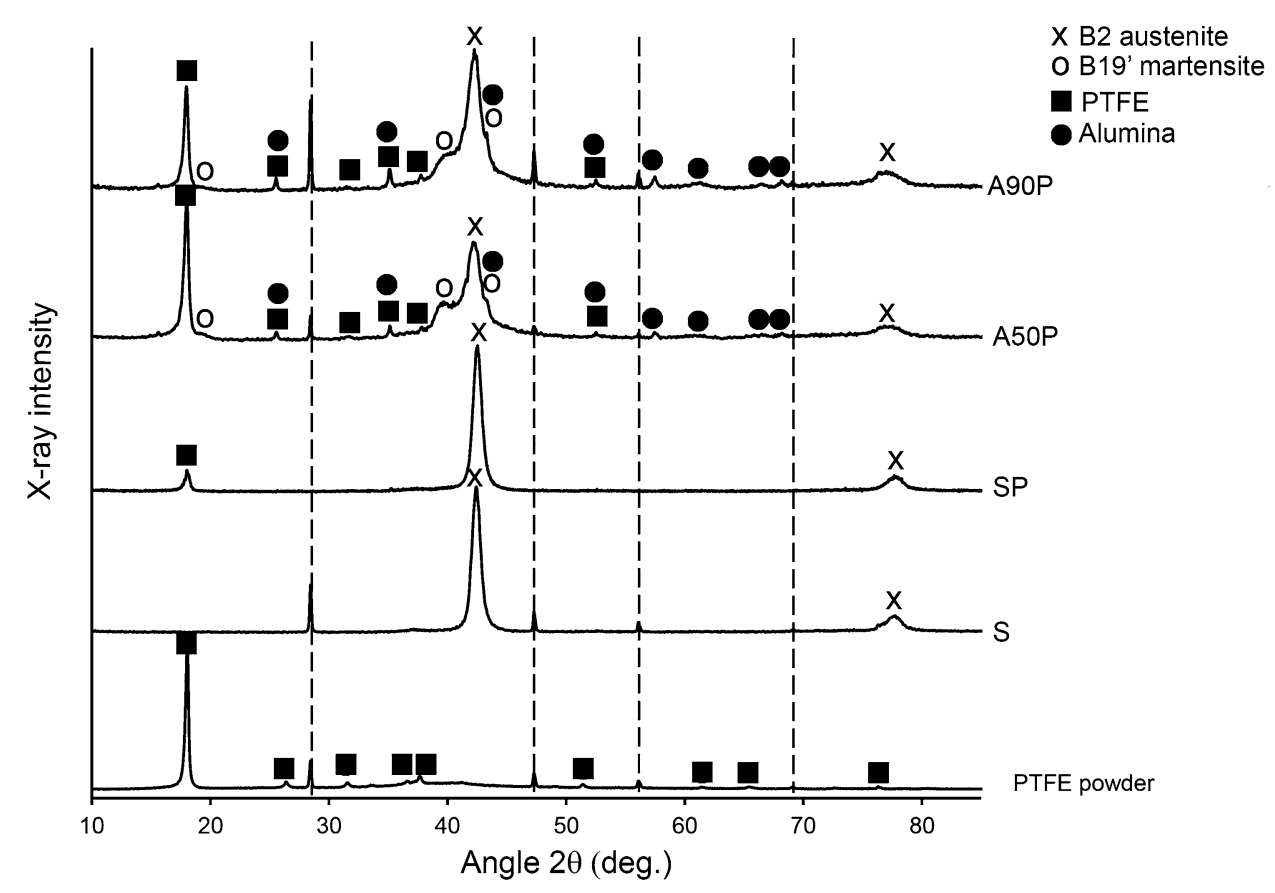




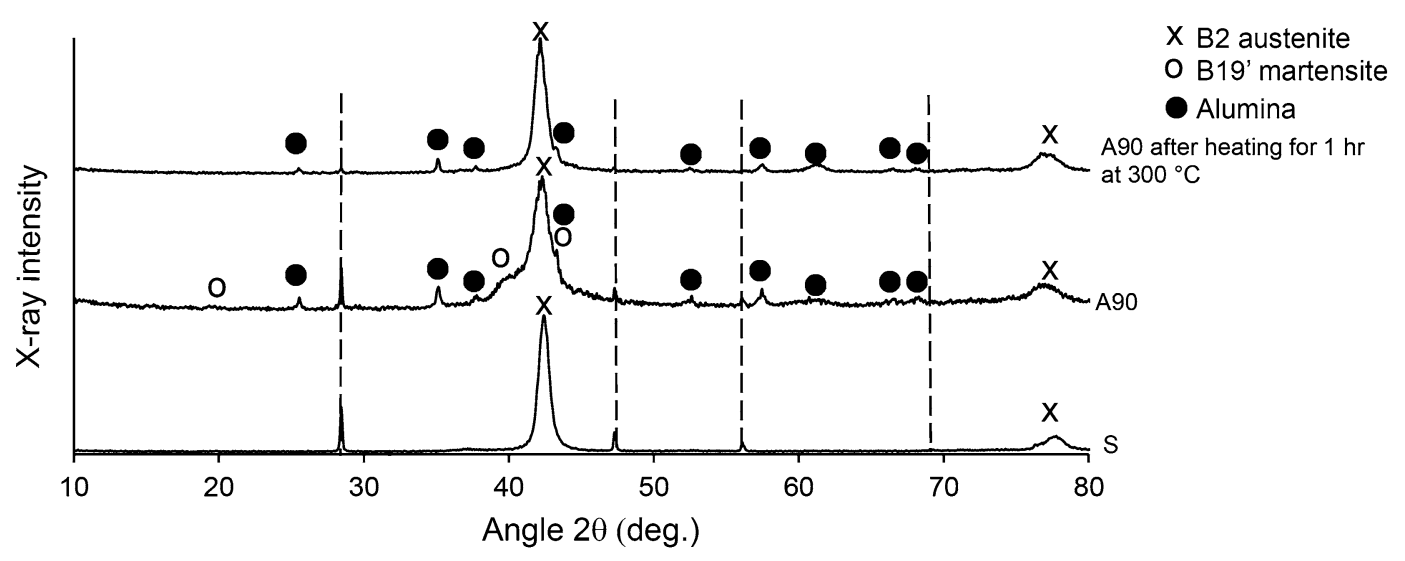

Fig. 4 XRD patterns of the S, A90 and A90 heated to $300{ }^{\circ} \mathrm{C}$ for $1 \mathrm{~h}$

$S$

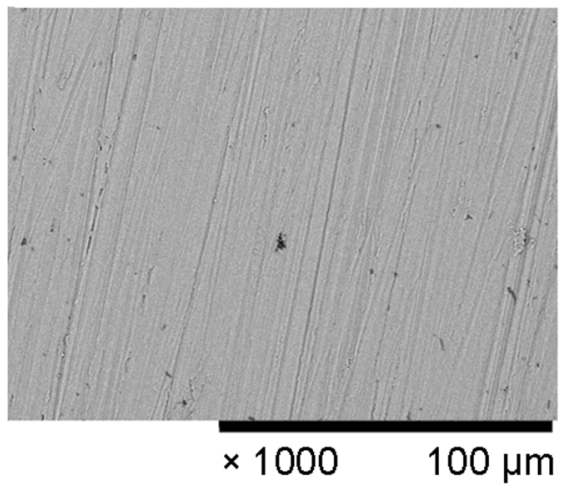

SP

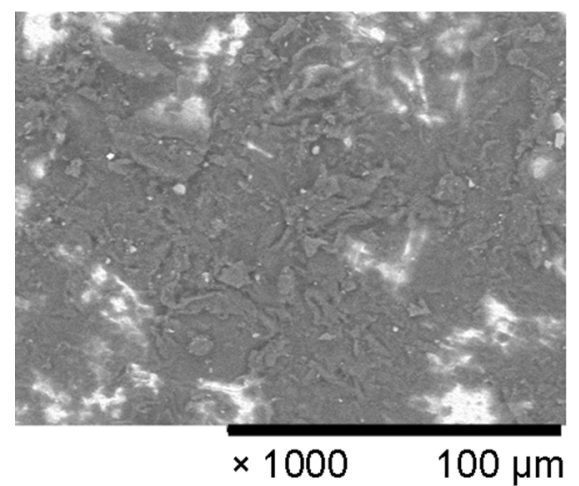

A50P

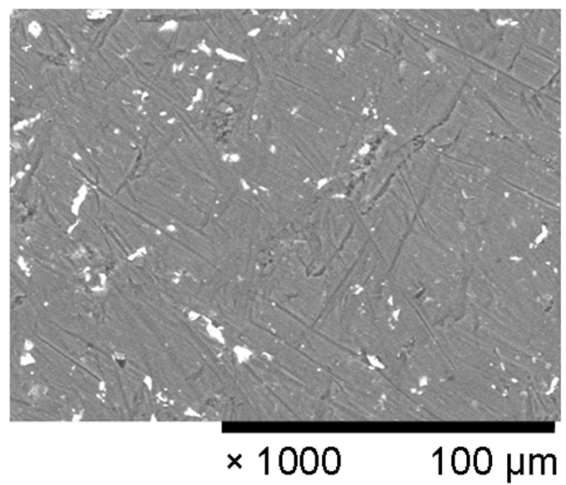

A50

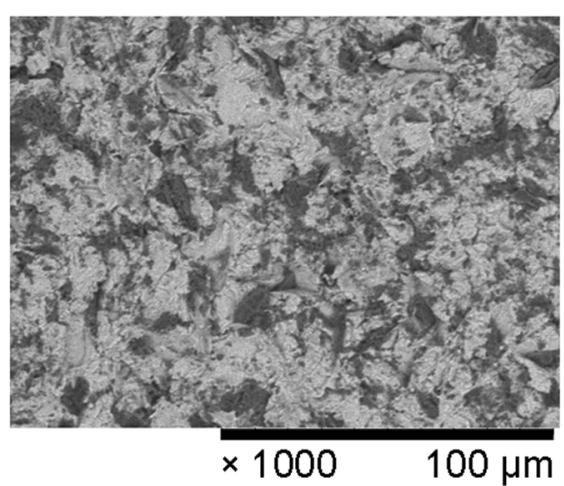

A90P

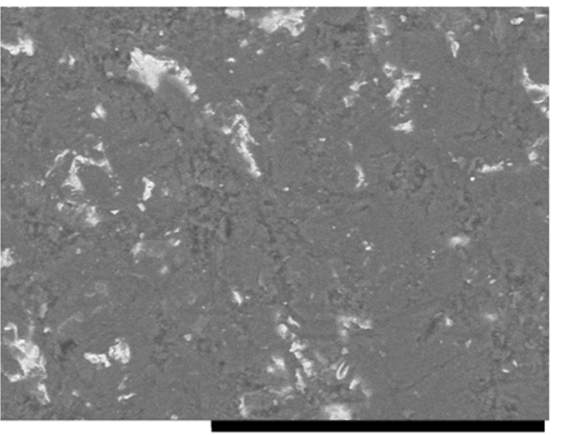

$\times 1000$

$100 \mu \mathrm{m}$

A90

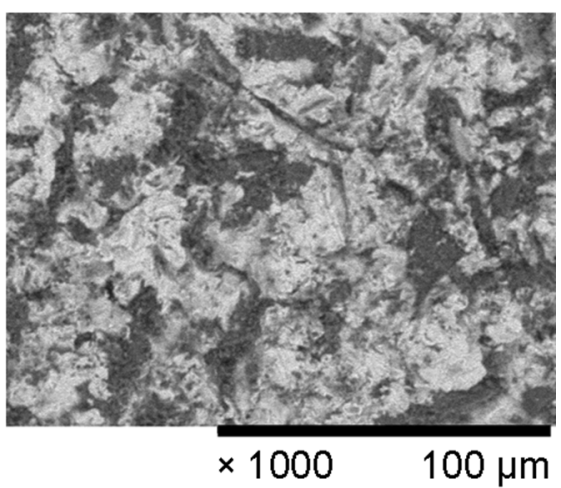

Fig. 5 SEM images of the surface of the as-supplied NiTi coupon and the treated samples

appear well coated with little of the underlying NiTi visible, confirming the good coating coverage observed in the cross-sections. The SP sample exhibits a relatively wellcoated surface however there appears to be more NiTi visible compared to the A50P and A90P substrates. The A50 and A90 samples exhibit clear tears and scratches. These features are more evident on the coupons blasted with the $<90 \mu \mathrm{m} \mathrm{Al}_{2} \mathrm{O}_{3}$ than the $<50 \mu \mathrm{m} \mathrm{Al}{ }_{2} \mathrm{O}_{3}$ due to the greater abrasion by the larger particle.
The average surface roughness of the samples after the surface modification are shown in Fig. 6. The average surface roughness $\left(R_{\mathrm{a}}\right)$ of each of the samples increased after surface modification compared to the unmodified titanium. One-way analysis of variance (ANOVA) was performed to determine whether the difference in roughness between the various substrates was statistically significant. The test yielded a $P$ value $=0.000$. This indicates that the roughness was significantly influenced by the type 


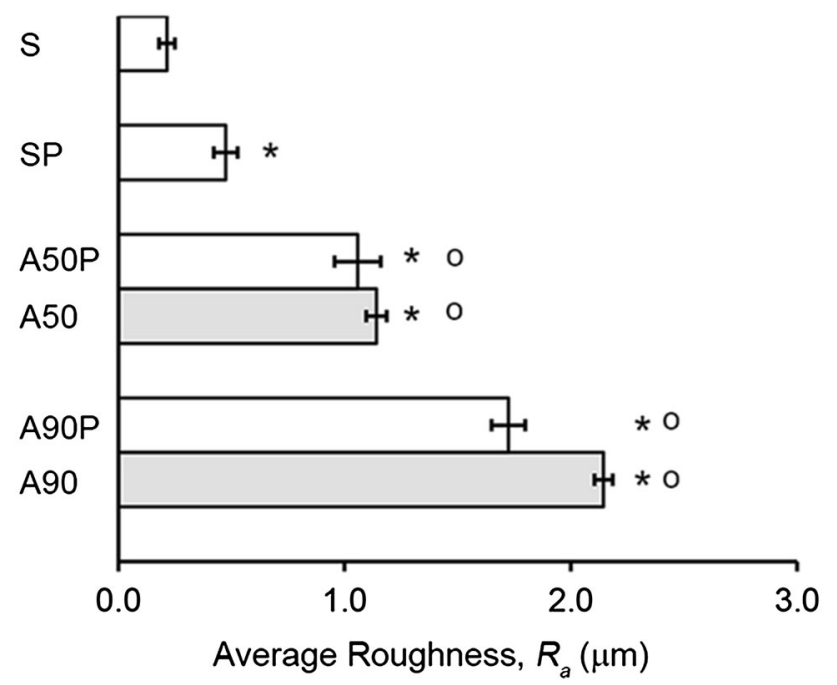

Fig. 6 Surface roughness of the substrates. $* P<0.05$ compared to $\mathrm{S}$; ${ }^{\mathrm{O}} P<0.05$ compared to SP

of blast medium used. Each of the coated surfaces had a significantly different roughness compared to the as-supplied indicating that the coating process significantly alters the surface roughness.

Cross-sections of the coupons and wires are shown in Figs. 7 and 8 respectively. A50P and A90P appear well coated with a continuous coating across the surface with a coating thickness $\approx 9 \mu \mathrm{m}$. The SP coating is not continuous and significantly thinner than the A50P and A90P coatings with a thickness $\approx 3-4 \mu \mathrm{m}$. The difference in coating thickness and coverage may be attributed to the chemical and mechanical bond formed between the coating and the substrate by the CoBlast process. The presence of the abrasive acts to remove the oxide layer exposing the reactive metal while mechanical stresses fragment the PTFE molecular chain, allowing carbon-metal bonding to occur. The high bond strength of PTFE to the metal due to fragmentation of the PTFE molecular chain due to mechanical stresses and carbon-metal bonding formation has previously been identified [18]. Secondary bonding between the $\mathrm{F}^{-}$ions and the metal $\mathrm{M}^{+}$ions may also contribute to the bond strength. Figures 7 and 8 show the coating penetrating into deep, narrow features of the A50P and A90P substrates as a result of the simultaneous deposition and abrasion. Conversely, the SP substrate does not form a chemical bond with substrate and is only weakly mechanically interlocked to the oxide layer at the surface. The A50 and $\mathrm{A} 90$ exhibit particles of $\mathrm{Al}_{2} \mathrm{O}_{3}$ embedded in the surface.

Figure 9 shows the wear tracks on each of the surfaces after 5000 cycles. Figure 10 shows the wear loss $\left(\mathrm{mm}^{3}\right)$ of the samples after 5000 cycles. The CoBlast samples appear to offer the greatest resistance to wear. There does not appear to be a significant difference between the A50P and A90P samples. The SP does appear to offer some resistance to wear compared to the S, A50 and A90 samples. However, it is not as effective as the CoBlast samples. Pure PTFE has low wear resistance, so the PTFE only coating is
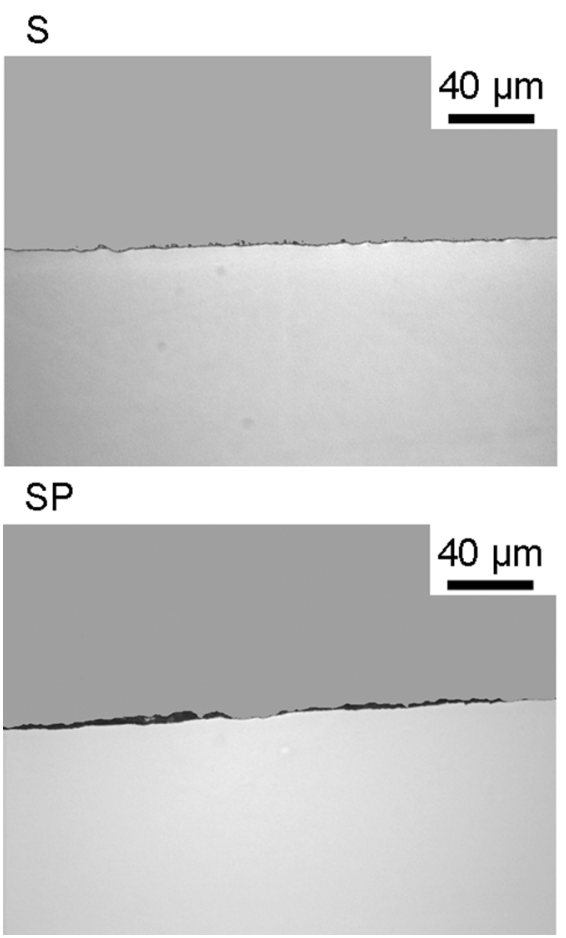

A50P

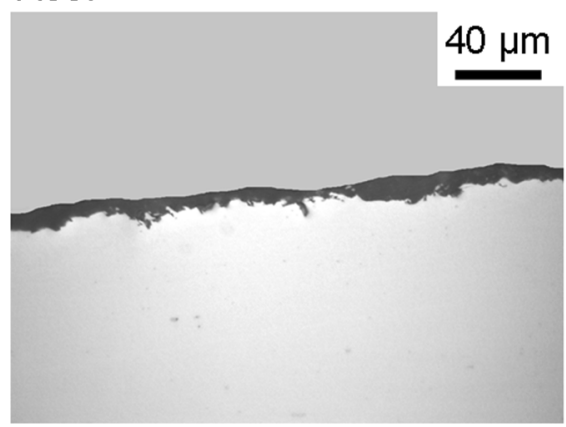

A50

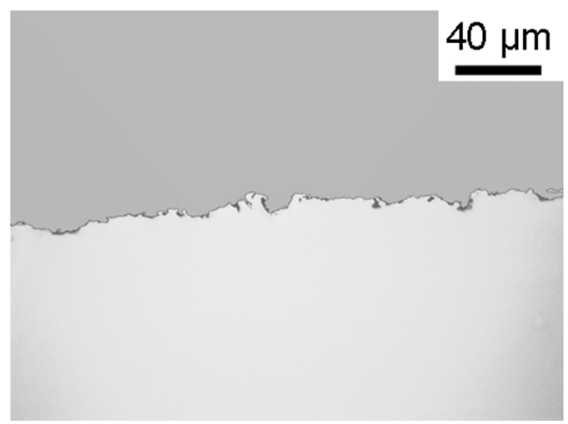

A90P

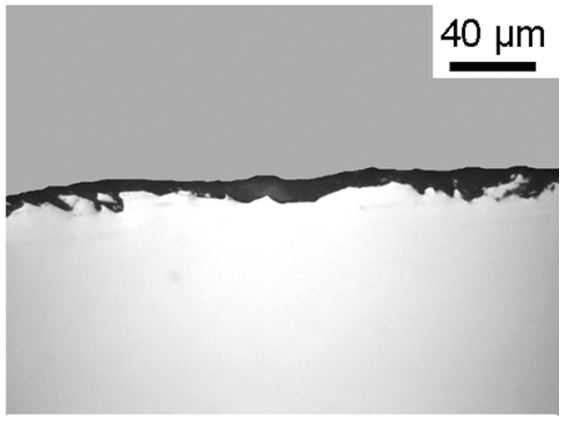

A90

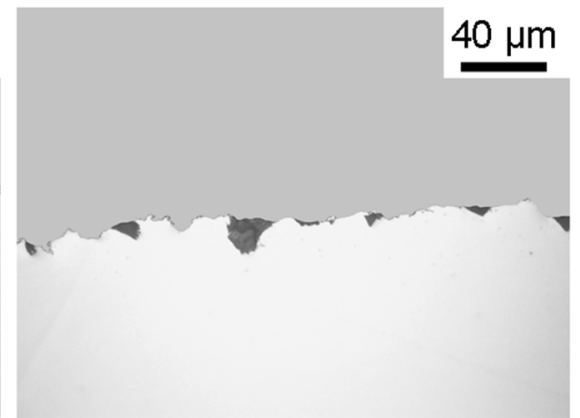

Fig. 7 Cross-sections of the coupons: S, SP, A50P, A90P, A50 and A90 


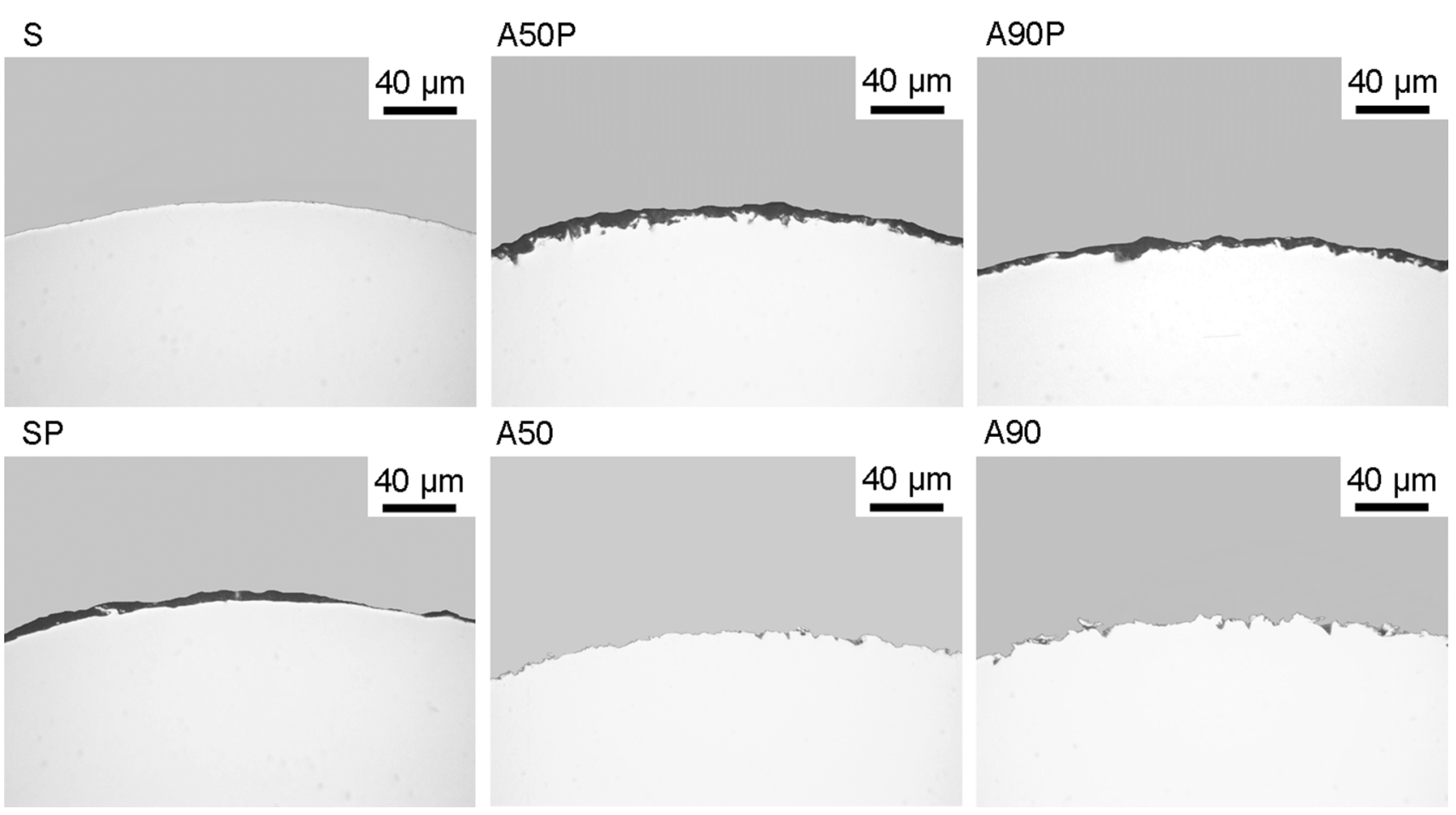

Fig. 8 Cross-sections of the wires: S, SP, A50P, A90P, A50 and A90
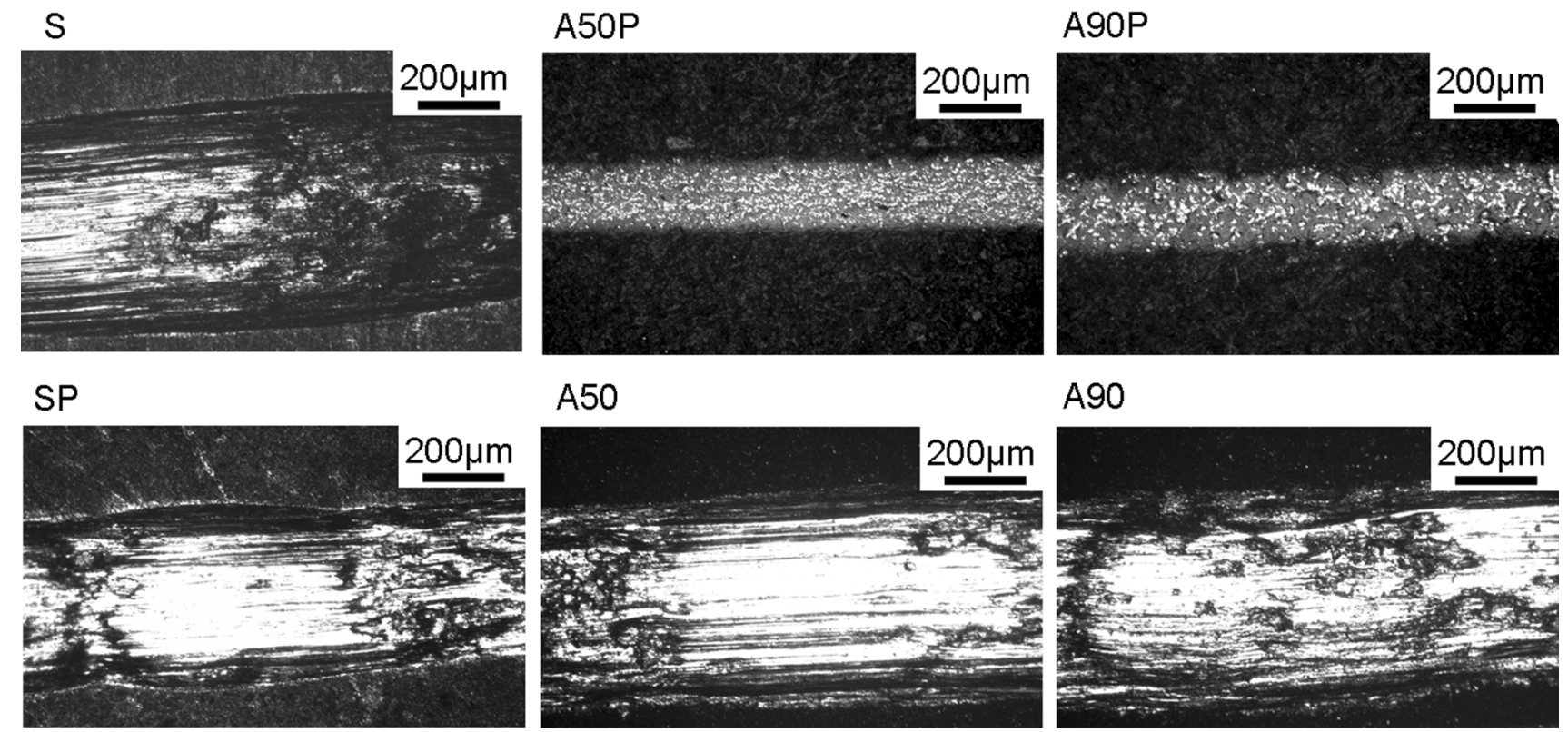

Fig. 9 Wear tracks on the surface of the coupons: S, SP, A50P, A90P, A50 and A90

quickly worn away to expose bare metal. The wear tracks of the CoBlast samples show a combination of metal (bright, reflective) and PTFE (dark, unreflective) areas. This suggests that the surface acts like a metal/PTFE composite [29], where the metal provides mechanical strength and the PTFE constantly lubricates the metal surface. The PTFE cannot be completely removed because it is deeply embedded within the metal by the simultaneous deposition and abrasion that is unique to the CoBlast technique. 
Fig. 10 Wear loss $\left(\mathrm{mm}^{3}\right)$ on the coupons. Error bars indicate standard deviation

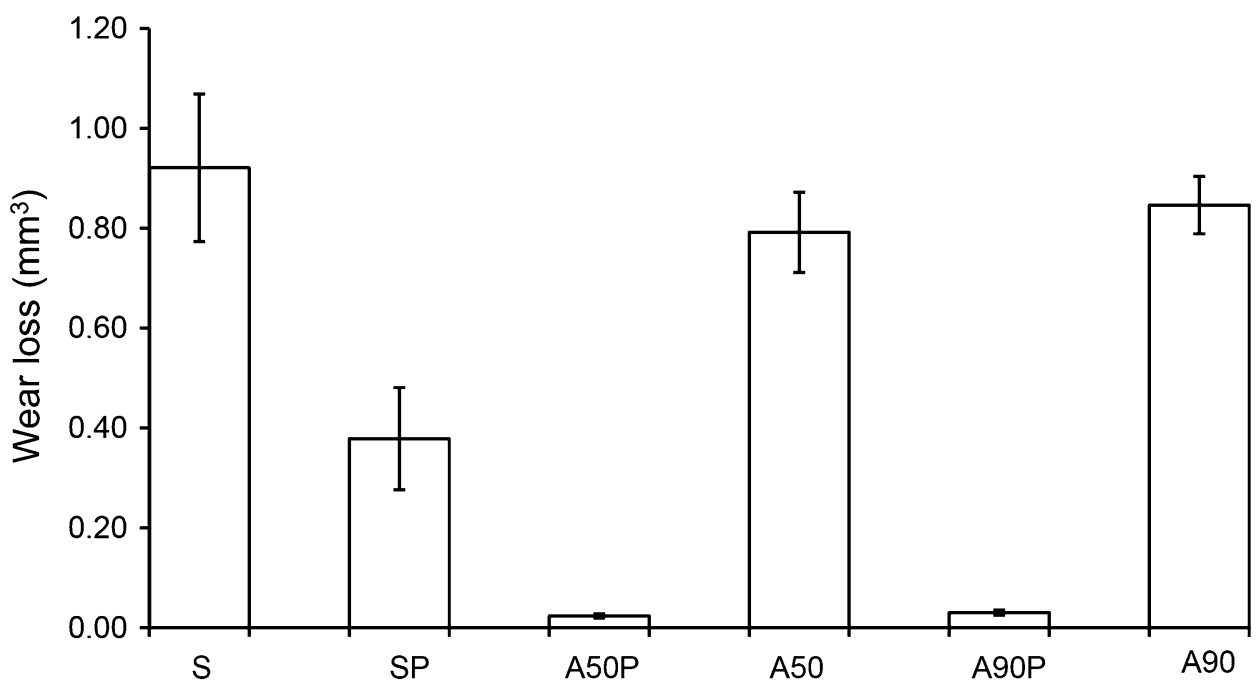

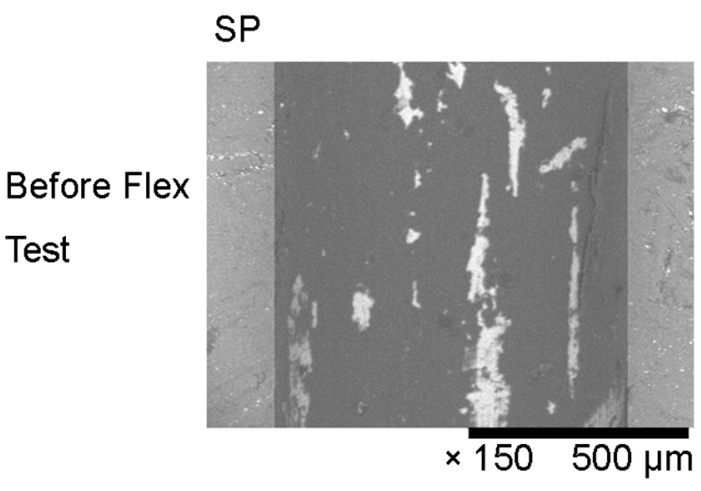

A50P
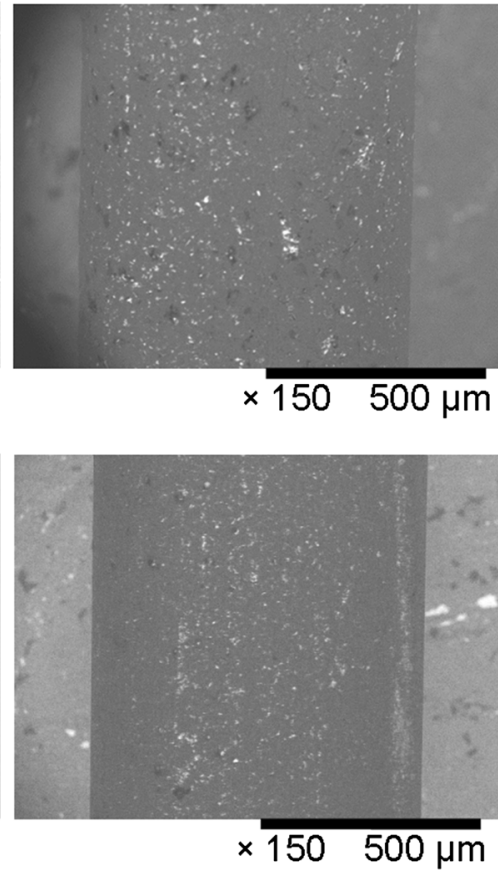

A90P
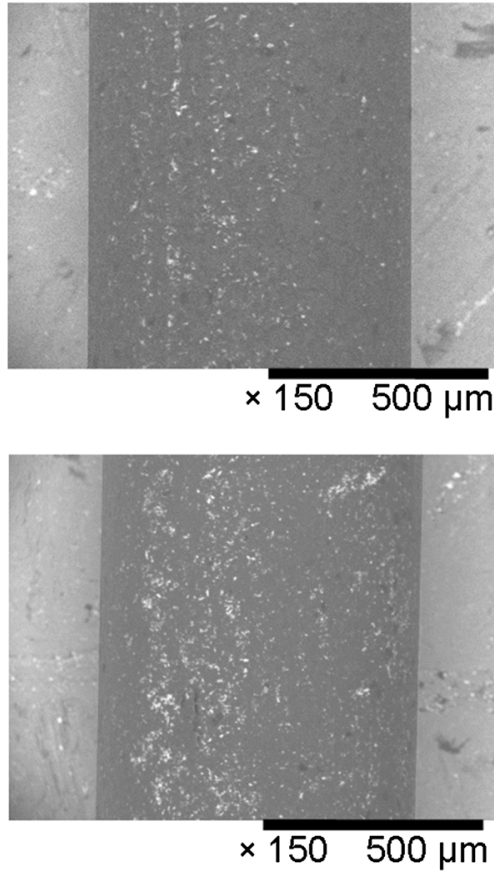

Fig. 11 Surface of the wires before and after fracture and flex testing

Figure 11 shows the surface of the wires after both fracture and flex testing. Visual examination of the wire surfaces after flex and fracture testing revealed no flaking of the CoBlast PTFE coatings, indicating a strongly bonded and flexible coating. In contrast, the substrate prepared by blasting with just PTFE was obviously damaged by the tests. No cracking of the substrate was observed for any of the wires, and a similar level of permanent deformation occurred in each sample, indicating that the superelasticity of the NiTi is not reduced by the blasting process.

\section{Conclusions}

CoBlasting PTFE onto a superelastic NiTi alloy can produce a well-adhered coating at ambient temperatures eliminates the need for pre- or post-processing steps such as cleaning, roughening, priming, baking or curing. The coating process offers enhanced wear compared to coating with PTFE only. The process does not adversely affect the mechanical properties of the superelastic NiTi wire. 
Acknowledgments This research is funded under the Programme for Research in Third-Level Institutions and co-funded under the European Regional Development Fund (ERDF).

\section{References}

1. Es-Souni M, Es-Souni M, Fischer-Brandies H (2002) On the properties of two binary NiTi shape memory alloys. effects of surface finish on the corrosion behaviour and in vitro biocompatibility. Biomaterials 23:2887-2894

2. Hasebe $\mathrm{T}$ et al (2006) Lubrication performance of diamond-like carbon and fluorinated diamond-like carbon coatings for intravascular guidewires. Diam Relat Mater 15:129-132

3. Liguori $\mathrm{G}$ et al (2008) Comparative experimental evaluation of guidewire use in urology. Urology 72:286-289

4. Clayman $\mathrm{M}$ et al (2004) Comparison of guide wires in urology. which, when and why? J Urol 171:2146-2150

5. Collier JP et al (1993) Loss of hydroxylapatite coating on retrieved total hip components. J Arthroplasty 8:389-393

6. Cook SD, Thomas KA, Kay JF (1991) Experimental coating defects in hydroxylapatite-coated implants. Clin Orthop Relat Res 265:280-290

7. Dunne CF et al (2015) Biological response to hydroxyapatite and fluorapatite coated dental screws. J Mater Sci. doi:10.1007/ s10856-014-5347-5

8. Dunne CF, Twomey B, O'Neill L, Stanton KT (2014) Co-blasting of titanium surfaces with an abrasive and hydroxyapatite to produce bioactive coatings: substrate and coating characterisation. J Biomater Appl 28:767-778

9. O'Neill L et al (2009) Deposition of substituted apatites onto titanium surfaces using a novel blasting process. Surf Coat Technol 204(4):484-488

10. O'Sullivan C et al (2010) Deposition of substituted apatites with anticolonizing properties onto titanium surfaces using a novel blasting process. J Biomed Mater Res B Appl Biomater 95B(1):141-149

11. O'Hare P et al (2010) Biological responses to hydroxyapatite surfaces deposited via a co-incident microblasting technique. Biomaterials 31(3):515-522

12. Prevy PS (2000) X-Ray diffraction characterization of crystallinity and phase composition in plasma-sprayed hydroxyapatite coatings. J Therm Spray Technol 9(3):369-376

13. Sobieszczyk S, Zielinski A (2008) Coatings in arthroplasty: review paper. Adv Mater Sci 8(4):35-54
14. Tsui YC, Doyle C, Clyne TW (1998) Plasma sprayed hydroxyapatite coatings on titanium substrates part 2: optimisation of coating properties. Biomaterials 19(22):2031-2044

15. Barry JN et al (2013) Evaluation and comparison of hydroxyapatite coatings deposited using both thermal and non-thermal techniques. Surf Coat Technol 226:82-91

16. Byrne GD, O’Neill L, Twomey B, Dowling DP (2013) Comparison between shot peening and abrasive blasting processes as deposition methods for hydroxyapatite coatings onto a titanium alloy. Surf Coat Technol 216:224-231

17. O'Sullivan C et al (2011) A modified surface on titanium deposited by a blasting process. Coatings 1(1):53-71

18. Biswas SK, Vijayan Kalyani (1992) Friction and wear of PTFEa review. Wear 158:193-211

19. Jiang XP et al (2006) Enhancement of fatigue and corrosion properties of pure $\mathrm{Ti}$ by sandblasting. Mater Sci Eng 429(1-2):30-35

20. Teoh SH (2000) Fatigue of biomaterials: a review. Int J Fatigue 22(10):825-837

21. Ewert JC, Böhm I, Peter R, Haider F (1997) The role of the martensite transformation for the mechanical amorphisation of NiTi. Acta Mater 45:2197-2206

22. Grant DM, Green SM, Wood JV (1995) The surface performance of shot peened and ion implanted NiTi shape memory alloy. Acta Metall Mater 43:1045-1051

23. Karaman I, Ersin Karaca H, Luo ZP, Maier HJ (2003) The effect of severe marforming on shape memory characteristics of a Tirich NiTi alloy processed using equal channel angular extrusion. Metall Mater Trans A 34:2527-2539

24. Koike DM Jr, Parkin, Nastasi M (1990) Crystal-to-amorphous transformation of NiTi induced by cold rolling. J Mater Res 5:1414-1418

25. Liao, Yiliang et al (2012) Deformation induced martensite in $\mathrm{NiTi}$ and its shape memory effects generated by low temperature laser shock peening. J Appl Phys 112(3):033515

26. Peterlechner M, Waitz T, Karnthaler HP (2009) Nanoscale amorphization of severely deformed NiTi shape memory alloys. Scripta Mater 60:1137-1140

27. Montross CS et al (2002) Laser shock processing and its effects on microstructure and properties of metal alloys: a review. Int $\mathbf{J}$ Fatigue 24:1021-1036

28. Liu Y, Tan G, Miyazaki S (2006) Deformation-induced martensite stabilisation in [100] single-crystalline Ni-Ti. Mater Sci Eng 438:612-616

29. Jones S, Stolarski TA, Tobe S (2004) Sliding performance of binary metal-PTFE coatings. Wear 257:539-554 